\title{
PROCESS AND SYSTEMS The systems approach at the sharp end
}

\author{
Author: Stephen Richard Harrold Cross
}

\section{A systems-based approach to patient safety has been} widely accepted and advocated. Despite this, and a renewed emphasis on organisational learning in improving the safety of patients, many patients still suffer unintended harm during their care. This paper reviews the central theoretical themes of a systems-based approach and raises four key questions relating for clinicians working at the 'sharp end': how can clinical teams best apply a systems approach to patient safety, how can patient safety move from a focus on past harm to a proactive approach, how can we avoid a corrosive culture of blame, and what should our response at the sharp end be to seemingly unsolvable problems?

KEYWORDS: Patient safety, systems approach

\section{Background}

The way in which a system operates is a major determinant of risk and risk can lead to harm. This simple concept, which underlies safety improvements in other industries, encapsulates the systems approach to patient safety. In this article, we rehearse some key concepts in system thinking and review some of the recent history of systems thinking in patient safety. But our perspective in these discussions is pragmatic: we aim to identify key issues for clinical practice at the sharp end and ask what has been the impact of systems thinking for clinicians working to prevent harm in their specialties.

\section{Patient safety is a continuing problem}

Patients are harmed every day in our healthcare systems. Wrong medicines are given to patients, the right medicine is given in the wrong dose, treatments are omitted or delayed, patients in hospital beds develop pressure ulcers or fall and break their femurs, otherwise diligent and capable clinicians unaccountably leave objects inside patients after operations, and sadly, many patients die in the care of doctors and nurses.

How many patients are harmed or die? Estimates vary but it is generally agreed that, for example, approximately $10 \%$ of hospital admissions result in an 'adverse event' where a patient is harmed. ${ }^{1}$ Around half of these are thought to be avoidable. And

Author: associate, The Academy of Medical Royal Colleges, London, UK yet, almost a decade and a half ago, we knew that medical care was problematic and this led to the publication of a key document in the UK. ${ }^{2}$ These documents recognised the importance of the systems in which patient care takes place and so introduced systems thinking to healthcare.

The safety improvement methodology that was developed in response was reactive: to look at what had gone wrong for patients and try to stop it happening again. The systems context was implicit in the key tool emerging from this initiative, root cause analysis (RCA), and incident investigations were expected to take into account systems factors such as equipment, training, supervision and so on. When things go wrong, clinicians have a duty to respond and improve; however, despite this focus on findand-fix, patient harm has not been prevented.

\section{Systems thinking}

All things exist in systems. In healthcare, individual system components include the patient, the clinicians, the equipment and the environment in which the care takes place. Systems thinking is a process of understanding the interrelatedness of the system components as part of a common process with a common purpose. In many public services, including healthcare, this requires focusing not only on the care of the service user but also on the many systems that support and enable clinical care.

Thinking in terms of systems factors when things go wrong for a patient, especially when there seem to be failings in professional competence or vigilance, can be difficult. In many industries we have often tended to assign personal blame to those actually in the critical situation - the last person to touch the controls of an aircraft or, in the case of healthcare, perhaps the last person to treat the patient. In the sections below, we explore the key differences between what has been called a person-based approach and a more mature systems-based approach.

\section{The person-based approach}

When an accident, adverse event or lapse in quality occurs it is natural to try and trace the cause and ask why and how it happened. In general, this has two aims: to learn where in the process the error occurred, and to judge whether any individuals were responsible and need to be held individually accountable. In this context, by far the most common allocation of cause is 'human error'. Implicit in this concept is the assumption that of many causal factors in what is sometimes a long chain of events, the action or inaction of a small number of human beings is of crucial significance. What generally follows is a focus on 
punishment or reward, the reduction of the role of humans in systems as far as may be possible, and an increase in 'command and control'. Blame, which seems to be a natural consequence of the identification of human error, frequently follows.

\section{The systems approach}

Healthcare systems are highly complex and can interact in unexpected ways. Our understanding of healthcare systems in recent years has moved from a person-centred approach towards a view that recognises that errors are more often consequences rather than causes. ${ }^{3}$ Performance is shaped by local circumstances, by the nature of the task and the tools, equipment and environment in which the activity is carried out. In this model, the human being is only one factor among many and the final outcome is a product of the interaction of many factors. The systems approach therefore views causality in a different way. It demands a more nuanced explanation where the goal is to understand why an event occurs through building up knowledge of how errors are affected by elements of the system. ${ }^{4}$

\section{The model of organisational accidents}

Person-centred views of safety or quality events often reflect the so-called 'domino theory' where human error triggers a following error, and then a subsequent error and so on until the event occurs: a row of dominos falling down, fully determined and with an easily identifiable initiating step in the chain. ${ }^{5}$

In place of the domino metaphor, the dominant metaphor in systems thinking in healthcare has become the 'Swiss cheese' model, ${ }^{6}$ introduced by James Reason to illustrate his 'model of organisational accidents'. This describes a system as possessing a series of layers of defence that prevent error. In an ideal world none of these defences would be breached; in reality the defences might resemble slices of Swiss cheese and have many 'holes' or routes through which failures may propagate. When all the holes in the series of slices become aligned, defences in depth have failed and an incident occurs. Part of this model is the distinction between active failures, which may be thought of as unsafe acts committed by people directly in contact with the patient, and latent failures which arise from organisational states resulting ultimately from decisions made by procedure writers, designers, administrators and management.

\section{Other systems approaches}

There have been a number of other approaches to systems thinking in patient safety, which have included other models of accident causation. The Swiss cheese model is often regarded as a linear model which assumes that a latent failure is a constant condition and therefore accessible to change. This may be misleading in practice, since systems factors vary constantly; the level of resource may be sufficient at times and at other times not - even varying within a day, a shift or an hour. Levels of training may be excellent or up-to-date in one ward and have only begun in another. In other models of accident causation, adverse events are seen as the result of a conjunction of a number of different factors. ${ }^{7}$ It was, for example, a lack of adequate supervision, combined with the violation of procedure, combined with the desire to deliver a care package, combined with the lack of reliable communication and checking which allowed the intrathecal administration of an incorrect drug in the most wellknown of medical adverse events, the vincristine incident. ${ }^{8}$

More recently, systems thinking in healthcare has encompassed 'human factors', a hybrid discipline encompassing elements of psychology, social psychology risk and ergonomics. This discipline had been central in building safety in other safety critical industries and many practical approaches have been promoted in healthcare. ${ }^{9}$ Human factors is often understood as the set of non-technical skills that underpin safe human performance (such as communication, situational awareness, decision making and leadership) but there is another aspect of human factors which focuses on the identification of risk within a system and the development of interventions and strategies to eliminate or reduce mitigate risk. These two aspects of human factors are sometimes referred to as 'person-based human factors' and 'system-based human factors'.

Literature on the systems approach has been dominated by work on human error, frameworks for risk and safety and incident reporting, perhaps the most significant work being the construction of a practical framework for safety produced by Vincent and colleagues as part of the programme in patient safety supported by The Health Foundation, The Measurement and Monitoring of Patient Safety. ${ }^{10}$ This provides a pragmatic approach toward creating patient safety based on five dimensions: learning from past harm; reliability of operations, processes and systems; sensitivity to operations (one of the key characteristics of highly reliable organisations in other sectors); integration of and learning from patient safety information; and, crucially, anticipation and preparedness, allowing a proactive response to future safety problems.

Other work has focused on a 'sector-wide' systems approach, where the consequences of the complexity and cross-influence of healthcare systems is recognised, ${ }^{11}$ and also on the need to understand the depth of complexity in systems, together with the factors that enable and resist change and improvement. ${ }^{12}$ Finally, work emerging from resilience engineering has suggested the need to move from 'safety I', where the approach to systems safety is based on reducing error - a state where as few things as possible go wrong - to 'safety II', where the system succeeds under varying conditions, and the adaptability and creativity of human performance in particular is seen as more critical than the prevention of error in a rigid system. ${ }^{13}$

\section{Systems thinking in practice}

The field of systems thinking in patient safety is wide-ranging and intellectually fascinating but it may be said that 'There is no silver bullet for patient safety. There are no short cuts, no quick fixes. ${ }^{14}$ Experience at the sharp end of clinical practice bears this out and is illustrated by an evaluation of a major patient safety initiative, again sponsored by The Health Foundation, Safer Clinical Systems, to which the author contributed, that some problems identified by safety teams seem insoluble, no matter how committed the team or how ingenious the approach. These larger problems need more resource, policy development or other interventions than can be easily delivered by working clinicians.

In this context, we would raise four key questions relating to the systems approach at the sharp end of patient care.

\footnotetext{
$>$ How can clinical teams best apply a systems approach to patient safety?
} 
> How can patient safety move from a reactive approach to the proactive prevention of harm?

> How can patient safety teams avoid a corrosive culture of blame?

> What should the response at the sharp end be to seemingly unsolvable problems?

\section{The systems approach at the sharp end}

Healthcare takes place in a primarily reactive culture. The major national initiatives in creating patient safety have emerged from An Organisation with a Memory and the more recent $A$ Promise to Learn - A Commitment to Act. ${ }^{15}$ Both exhort clinicians and managers to use incidents of harm and near-misses to learn about safety failures and improve practice and the systems that support practice. Although not described formally as such, this comprises a 'Safety management system'. Put simply, there was an expectation that it would work as follows: in a culture which eschewed individual blame and accepted that, by and large, clinicians intended to do their best for patients, errors and adverse events should be reported. After reporting through local risk-management systems, harmful events as well as 'nearmisses' (which might indicate future risks) were to be investigated thoroughly. In response to this investigation we would change the way we worked and become safer.

In response to this concept, the NHS has created local reporting systems, a National Reporting and Learning System which tries to aggregate, measure and share safety issues, and a regulatory commitment to the analysis of adverse events where significant harm to a patient has occurred. In a hospital trust, it is not uncommon for 1,000 incidents to be reported every month, for example, and for 10 RCAs per month to be carried out. Since each of these RCAs will typically contain many discrete recommendations for improvement, the volume of effort nationally can be readily appreciated.

For most clinicians, this is where their chief interaction with patient safety takes place and where they might be expected to incorporate a knowledge of systems and how they may be improved or made safer. Sadly, this rarely works as planned. As part of the 'Safer clinical systems' programme, we used several tools to assess the culture of safety, including the Manchester Patient Safety Framework: ${ }^{16}$ all pointed to a safety culture dominated by a 'bureaucratic' response to adverse events, where paperwork takes precedence over the management of error and contextual risk, where systems factors were usually unaddressed and the blame of individual members of staff was common. This culture, which can be encouraged by the cycle of deadlines required by commissioners, does not easily lead to systems change. Investigations often ignore or downplay systems factors, result in poor-quality recommendations for change and perpetuate a culture of blame. Most importantly, however, the entire process is only kicked into activity after a patient has already been harmed; it is an entirely reactive approach to safety.

Where else are clinicians able to apply a systems approach? In theory, adopting a framework for measurement and monitoring of safety should support systems change. However, in reality, the very heart of the systems approach to safety - the management of risk in clinical systems - receives patchy attention. At this point, a brief outline of some of the findings of the Safer clinical systems ${ }^{17}$ programme is highly relevant. This programme, which took place in eight acute trusts in the UK and one follow-up trust, took an explicitly systems-based approach to safety, based in part on familiar and successful practices from other safety-critical industries. Although 'person-centred' human factors, focusing on the non-technical skills such as communication and situational awareness, was part of the programme, the main design element was the proactive identification of risk in clinical systems using mapping techniques and 'Failure mode and effects analysis' (FMEA). (FMEA is a systematic method used in many industries where task risks are identified and evaluated.) Clinical teams took the outputs from proactive analyses to design and implement safety interventions directly related to the risks to patients that they had identified. The teams also used safety cases, which are frequently employed as tools to judge and manage safety proactively in high-risk industries. ${ }^{18}$ At its simplest, a safety case provides an analysis of risk based on a systematic mapping and proactive safety appraisal. In practice, they are used not only to record current knowledge of safety but also to highlight and measure the interventions needed to improve safety proactively and on the basis of evidence.

Taking a systems-based approach in a defined clinical area can be difficult and, from experience in risk management, is rarely carried out proactively in healthcare. Arguably, change in practice is most effective when it is driven from the sharp end, as opposed to "topdown' quality and safety drives, and where the tacit knowledge of all staff is unlocked to identify and manage risk. These qualities were integral to 'Safer clinical systems' mapping, risk evaluation and intervention design were mostly carried out in consensus groups, cutting across professional boundaries and the 'silos' created around specialties in complex organisations. Whatever the theories and high-level thinking about the systems approach tell us, to be effective in everyday experience, it has to be linked meaningfully to things that can be done at the sharp end.

This systems approach was evaluated in many ways. A formal evaluation conducted by skilled evaluators ${ }^{19}$ highlighted a sobering number of risks in the clinical areas concerned - many of them previously unknown to the clinical teams and representing future risks to patients. They commented on the utility of the techniques used in addressing them, changes in safety culture and, pithily, to the existence of 'big hairy problems' that could not be addressed locally; if there are insufficient numbers of pharmacists in a system to review every patient's medication daily (as if often the case), for example, the structural and budgetary implications may go far beyond the ability of local teams to fully solve.

However, the final reporting on the project revealed that a systems approach at the sharp had been effective at the core of safety management - reducing risk proactively. For all identified risks in the systems, teams reported a fall in the numbers of identified risks of $62 \%$; for those risks categorised as 'high', a reduction of $70 \% .{ }^{20}$ As importantly, the impact of a systems approach - carried out at the sharp end by multiprofessional teams - was evident in their reflections at the conclusion to the programme. For example, one participant working on safe handovers commented that,

We came into this work with a hypothesis of a reactive system. We thought we knew where the hazards were. We were incorrect. Our measurement has now shown that one in seven patients being handed over in a week is incorrectly handed over.

Overall, in both formal safety culture measurements and described experience, there was clear evidence of the practical and cultural changes at the sharp end from a systems-based approach. 


\section{Systems approach at the sharp end - taking stock}

Revisiting the key questions raised, I offer the following comments based on the 'Safer clinical systems' programme and my own experience in managing patient safety in a large hospital trust.

Firstly, how can clinical teams best apply a systems approach? It is worth noting that patient safety education for clinical staff, as reflected in a review of specialist educational programmes, is almost entirely related to the safety of direct interactions with patients - what might be termed 'clinical risk'. However, keeping patients safe depends on more than this - it also depends on managing the risks created by the systems clinicians work within, what might be termed 'system risk'. Although clinicians will carry out and take part in RCAs following harmful incidents, there is a clear need to ensure that these investigations and subsequent interventions encompass system risk. But more is needed: a systems approach to reducing risk through the adoption of mapping and FMEA has much to offer in this context and provides an explicit link between systems thinking and safety at the sharp end.

Secondly, how can patient safety move from a reactive approach to the proactive prevention of harm? The failure of organisations to be demonstrably aware of and in control of risk is a serious problem which would not be accepted in other industries. Here, the use of the techniques mentioned above, perhaps with the use of a working safety case, ensures that a proactive approach is applied.

Thirdly, how can patient safety teams avoid a corrosive culture of blame? The plausibility of finding individual error and placing this as the chief causal factor in safety incidents is hard to avoid after the event. But a proactive systems approach means that error traps and system risk can be addressed without individual blame. Taking a 'Just culture, ${ }^{21}$ approach to investigating clinical failures is undoubtedly helpful but, as high-profile cases have shown, the natural human desire for simple explanations and individual culpability will usually dominate; we need to address risk before patients are harmed. The creation of the Health Services Investigation Branch, announced by the secretary of state in July 2015 may have some potential in addressing blame culture but, unless it is able to truly embrace proactive approaches to risk, will still further a reactive approach to safety management.

Finally, what should the response at the sharp end be to seemingly unsolvable problems? What can frontline staff do when they identify significant residual risks as a result of this work that they feel cannot be changed - the big problems that are just too difficult to address? What if there is no resource to provide extra pharmacists? What if the organisation simply cannot locate and recruit or even afford the nurses needed for the night shifts? What if the senior managers in the organisation seek comfort and do not wish to seek out and confront problems honestly? Here, risk awareness and good governance is essential. Uncontrolled risks have to be managed appropriately. In most cases they will be placed on the risk register of the organisation - sometimes used wisely as a live document and improvement tool, but sometimes a repository of things we can do nothing about but continue to worry us. We believe that uncontrolled risk to patients should be escalated and reviewed honestly through systematic appraisal of risk, as was conducted in 'Safer clinical systems'. In most cases, a senior manager (probably an executive board member) must take the responsibility for how to deal with uncontrolled risks to patients. He or she may decide, for example, to simply transfer the risk to another trust and cease to offer the services concerned; they may decide that it is better to try and mitigate the risk through detailed resilience planning and day-to-day monitoring; or the organisation may simply decide to accept the risk. In those cases, the escalation of risk must go beyond the healthcare organisation concerned - to those who commission, regulate or fund.

\section{Conclusion}

The systems approach to safety has been essential in creating safety in high-risk industries and has been rightly advocated in the complex field of healthcare. However, systems exist at many levels and the working clinician may find it difficult to develop and apply a detailed understanding of system-based safety in local areas. There is a gap both in education and in resource; the sort of approach used in programmes like 'Safer clinical systems' requires a technical understanding of risk and intervention, and an ability to apply analytical tools, that goes beyond that usually available in trusts. Risk managers in NHS trusts will usually spend more time - or even all their time - managing the reporting and analysis processes, at the expense of seizing control of risk upfront.

Nevertheless, such a systems-based approach is necessary; patient safety incidents continue to occur, despite our current wholly reactive approach to managing safety. The tools and techniques required are more unfamiliar than demanding and can be easily applied, given the will and the support of the organisational culture. And perhaps that is the key lesson of the systems approach: a cultural change is needed in safety - but a change not limited to more or better retrospective investigations, a change in the direction of proactive, systems-based safety management. In this context, clinicians at the sharp end must be supported to make systems approaches accessible with practical tools in both reactive and proactive methods.

\section{References}

1 Vincent C, Neale G, Woloshnyowych M. Adverse events in British hospitals: preliminary retrospective record review. BMJ 2001;322:517-9.

2 Department of Health. An organisation with a memory. Report of an expert group on learning from adverse events in the NHS. The Stationary Office, 2000.

3 Reason J. Human Error. Cambridge University Press, 1990.

4 Cook RI, Woods DD, Miller C. A Tale of Two Stories: Contrasting Views of Patient Safety, Report from a Workshop on Assembling the Scientific Basis for Progress on Patient Safety. Washington, DC: National Patient Safety Foundation, 1998.

5 Heinrich HW. Industrial accident prevention: A scientific approach. New York: McGraw-Hill, 1931.

6 Reason J. Managing the risks of organisational accidents. Ashgate, 1997

7 Hollnagel E. Barriers and accident prevention. Ashgate, 2004.

8 Toft B. External inquiry into the adverse incident that occurred at Queens Medical Centre, Nottingham, 4th January 2001. Department of Health, 2001.

9 Carayon P, Wetterneck TB, Rivera-Rodriguez AJ et al. Human factors systems approach to healthcare quality and patient safety. Appl Ergon 2013;45:14-25.

10 Vincent C, Burnett S, Carthey J. The measurement and monitoring of safety. The Health Foundation, 2013.

11 Pronovost PJ, Ravitz AD, Stoll RA, Kennedy SB. Transforming Patient Safety. A sector-wide systems approach. Report of the WISH Patient Safety Forum. World Innovation Summit for Health, 2015. 
12 Braithwaite J. Changing how we think about healthcare improvement. BMJ 2018;361:k2014.

13 Hollnagel E, Wears RL, Braithwaite J. From Safety I to Safety II: A White Paper. The Resilient Health Care Net. Published simultaneously by the University of Southern Denmark, University of Florida, USA, and Macquarie University, Australia.

14 Ghebreyesus TD. 6th Annual World Patient Safety, Science \& Technology Summit. World Health Organization, 2018. www. who.int/dg/speeches/2018/patient-safety-summit/en/ [Accessed 16 July 2018].

15 National Advisory Group on the Safety of Patients in England. A promise to learn - a commitment to act. Improving the safety of patients in England. Crown Copyright, 2016.

16 Parker D, Kirk S, Claridge T, Lawrie M, Ashcroft DM. The Manchester Patient Safety Framework (MaPSaF). Patient Safety Research: shaping the European agenda - International Conference Porto, Portugal, 2007

17 Safer Clinical Systems, 2014. The Health Foundation. www.health. org.uk/programmes/safer-clinical-systems [Accessed 16 July 2018].
18 Sujan M, Spurgeon P, Cooke M et al. The development of safety cases for healthcare services: Practical experiences, opportunities and challenges. Reliability Engineering and System Safety 2015;140:200-7.

19 Dixon-Woods M, Martin G, Tarrant C et al. Safer Clinical Systems: evaluation findings. The Health Foundation, 2014.

20 Spurgeon P, Flanagan $\mathrm{H}$, Cooke $\mathrm{M}$ et al. Creating safer health systems: lessons from other sectors and an account of an application in the Safer Clinical Systems programme. Health Serv Manage Res 2017;30:85-93.

21 Dekker S. Just Culture: Balancing Safety and Accountability, 1st edn. Ashgate, 2007.

Address for correspondence: Dr Stephen Richard Harrold Cross, The Academy of Medical Royal Colleges, 10 Dallington Street, London EC1V 0DB, UK.

Email: stevecrossuk@gmail.com
'This landmark report lays out in the starkest terms yet the devastating impact air pollution is having on our health, our economy and our society as a whole.'

\section{Every breath we take The lifelong impact of air pollution}

This major report plainly sets out the dangerous impact that air pollution has on our nation's health. Compiled by experts in medicine and environmental sciences, the report discusses the current evidence and draws up recommendations for action.

ISBN 978-1-86016-567-2 £15 including $p+p$ or free to download

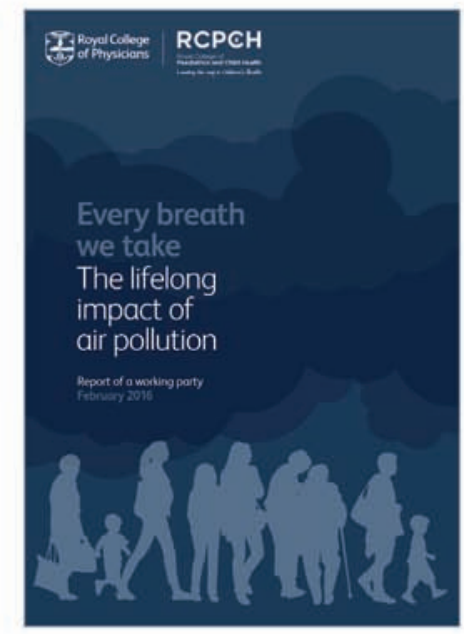

Order a copy: shop.rcplondon.ac.uk
Download the report: rcplondon.ac.uk/pollution

\section{Royal College of Physicians}

